\title{
Downregulation of long non-coding RNA nuclear enriched abundant transcript 1 promotes cell proliferation and inhibits cell apoptosis by targeting miR-193a in myocardial ischemia/reperfusion injury
}

\author{
Lingyun Ren ${ }^{1 \dagger}$, Shanshan Chen ${ }^{2+}$, Wei Liu', Pan Hou', Wei Sun ${ }^{1}$ and Hong Yan ${ }^{1 *}$ (D)
}

\begin{abstract}
Background: This study aimed to investigate the effect of long non-coding RNA nuclear enriched abundant transcript 1 (Inc-NEAT1) on cell proliferation and apoptosis in myocardial ischemia/reperfusion (I/R) injury cells, and explore its target miRNAs.

Methods: $\mathrm{H} 9 \mathrm{c} 2$ cells were cultured in oxygen and glucose deprivation followed by reperfusion (OGD/R) condition to construct a myocardial I/R injury model. Blank shRNA and Inc-NEAT1 shRNA were transferred into normal H9c2 cells and I/R injury H9c2 cells as Normal\&sh-NC, OGD/R\&sh-NC and OGD/R\&sh-NEAT1 groups. Rescue experiment was performed by transfection of NC inhibitor plasmids, miR-193a inhibitor plasmids and NEAT1 shRNA into I/R injury cardiocytes. RNA expression, cell proliferation and cell apoptosis rate were detected by qPCR, CCK-8 and AV/ PI respectively.

Results: After OGD/R induction, H9c2 cell apoptosis was greatly increased while cell proliferation was decreased, which indicated successful establishment of myocardial I/R injury model, and Inc-NEAT1 expression was elevated as well. Cell proliferation rate was increased in OGD/R\&sh-NEAT1 group at $48 \mathrm{~h}$ and $72 \mathrm{~h}$ compared to OGD/R\&sh-NC group, while cell apoptosis was reduced in OGC/R\&sh-NEAT1 group compared to OGD/R\&sh-NC group. Target miRNAs detection indicated the negative regulation of Inc-NEAT1 on miR-193a but not miR-182 or miR-141. In rescue experiment, downregulation of Inc-NEAT1 promoted cell proliferation and inhibited cell apoptosis through targeting miR-193a in I/R injury H9c2 cells.

Conclusion: Lnc-NEAT1 is overexpressed in myocardial I/R injury cells compared to normal myocardial cells, and downregulation of Inc-NEAT1 enhances cell proliferation while inhibits cell apoptosis through targeting miR-193a in I/R injury H9c2 cells.
\end{abstract}

Keywords: Long non-coding RNA nuclear enriched abundant transcript 1, Myocardial ischemia/reperfusion injury, miR-193a, Cell proliferation, Cell apoptosis

\footnotetext{
*Correspondence: shizikuang2@163.com

${ }^{\dagger}$ Lingyun Ren and Shanshan Chen contributed equally to this work.

'Department of Anesthesiology, The Central Hospital of Wuhan, Tongji

Medical College, Huazhong University of Science and Technology, 26,

Shengli Street, Jiang'an District, Wuhan 430014, China

Full list of author information is available at the end of the article
}

(c) The Author(s). 2019 Open Access This article is distributed under the terms of the Creative Commons Attribution 4.0 International License (http://creativecommons.org/licenses/by/4.0/), which permits unrestricted use, distribution, and reproduction in any medium, provided you give appropriate credit to the original author(s) and the source, provide a link to the Creative Commons license, and indicate if changes were made. The Creative Commons Public Domain Dedication waiver (http://creativecommons.org/publicdomain/zero/1.0/) applies to the data made available in this article, unless otherwise stated. 


\section{Background}

Cardiovascular disease, a class of diseases involving the heart or blood vessels, has been reported to be the leading cause of deaths worldwide, which is responsible for approximately 17.3 million deaths in 2013 around the world, and increases rate of deaths from 25 to $40 \%$ during the period from 1990 to 2010 in China [1-3]. Most of the cardiovascular disease patients would generate myocardial ischemia when blood flow to heart is decreased due to a partial or complete blockage of cardiovascular arteries, which reduces the acquisition of enough oxygen, damages heart muscle and causes serious abnormal heart rhythms [4]. For settling this problem, reperfusion is popularly utilized, which delivers oxygen and nutrients to cardiac myocytes, subsquently sustaining aerobic metabolism and ATP generation, while it may cause paradoxical cardiomyocyte dysfunction as well as result in tissue damage [5]. These additional damages are defined as myocardial ischemia/reperfusion (I/R) injury, which leads to worse cardiovascular outcomes, suc h as stroke, myocardial infarction or even sudden death [5-7]. Thus, exploring novel mechanisms of myocardial I/R injury is of great importance for its prevention and treatment.

Long non-coding RNAs (lncRNAs), a type of RNAs with over 200 nucleotides in length and characterized by no protein coding properties, emerge as crucial mediators in several biological processes (such as cell proliferation, cells differentiation as well as apoptosis), among which parts of these lncRNAs appear with dysregulated and devote into the development and progression of various diseases (particularly cardiovascular diseases) [8-11]. LncRNA nuclear enriched abundant transcript 1 (lnc-NEAT1) is transcribed by RNA polymerase II from a common promoter and widely expressed in mammalian cells, which functions as scaffolds of nuclear bodies [12-14]. Accumulating evidences have revealed that lnc-NEAT1 regulates cells activities through targeting multiple miRNAs or signaling pathways in several diseases including traumatic brain injury, diabetes mellitus as well as carcinomas [15-17]. Considering information about the underlying mechanism of lnc-NEAT1 in myocardial I/R injury is rarely known, we conducted this study to investigate the effect of lnc-NEAT1 on cell proliferation and apoptosis in myocardial I/R injury cells, and explore its target miRNAs.

\section{Methods}

\section{Cells culture}

Rat cardiac muscle cell line H9c2 was purchased from Cell Resource Center of Shanghai Institute of Life Sciences, Chinese Academy of Sciences (Shanghai, China), and H9c2 cells were cultured in 90\% Dulbecco's Modified Eagle's Medium (DMEM) medium (Gibco, USA) complimented with 10\% Fetal Bovine Serum (FBS) (Gibco, USA). Cells were cultured in an incubator with 95\% air and $5 \% \mathrm{CO}_{2}$. at $37{ }^{\circ} \mathrm{C}$.

\section{Construction of myocardial I/R injury model}

$\mathrm{H} 9 \mathrm{c} 2$ cells were cultured in oxygen and glucose deprivation followed by reperfusion (OGD/R) condition to construct a myocardial I/R injury model, and the detailed process was as follows: (1) H9c2 cells were firstly cultured in glucosefree DMEM (Gibco, USA) medium and incubated in oxygen-free atmosphere $\left(95 \% \mathrm{~N}_{2}\right.$ and $5 \% \mathrm{CO}_{2}$ at $\left.37^{\circ} \mathrm{C}\right)$ for 10 h; (2) cells were then cultured in normal DMEM (Gibco, USA) medium under normal atmosphere (95\% air and 5\% $\mathrm{CO}_{2}$.at $37^{\circ} \mathrm{C}$ ) for another $24 \mathrm{~h}$; (3) AV/PI was used to determine cell apoptosis rate to confirm the construction of myocardial I/R injury model; (4) qPCR was used to determine lnc-NEAT1 expression.

\section{Effect of Inc-NEAT1 shRNA on cell proliferation and apoptosis in myocardial I/R injury model}

Blank shRNA and lnc-NEAT1 shRNA plasmids were constructed by Shanghai QeeJen Bio-tech Institution (Shanghai, China), and then transferred into normal H9c2 cells and I/R injury H9c2 cells using HilyMax (Dojindo, Japan). Cells were divided into 3 groups according to different interventions as follows: (1) Normal\&sh-NC group (blank shRNA transferred into normal H9c2 cells); (2) OGD/R\&sh-NC group (blank shRNA transferred into I/R injury H9c2 cells); (3) OGD/ R\&sh-NEAT1 group (lnc-NEAT1 shRNA transferred into I/ $\mathrm{R}$ injury H9c2 cells). Subsequently, lnc-NEAT1 expression was detected by qPCR at $24 \mathrm{~h}$, cell proliferation was detected by CCK- 8 at $0 \mathrm{~h}, 24 \mathrm{~h}, 48 \mathrm{~h}$ and $72 \mathrm{~h}$, cell apoptosis rate was detected by AV/PI at $72 \mathrm{~h}$.

\section{Prediction and measurement of target miRNAs of Inc- NEAT1}

Target miRNAs of lnc-NEAT1 in I/R injury cardiocytes was predicted using starBase and miRcode database, and three miRNAs related to I/R injury were selected to be measured by qPCR in this study: miR-141, miR-182 and miR-193a.

\section{Effect of Lnc-NEAT1/miR-193a on cell proliferation and apoptosis in myocardial I/R injury model}

$\mathrm{I} / \mathrm{R}$ injury cardiocytes were then transferred by (1) NC inhibitor plasmids (NC-inhibitor group); (2) miR-193a inhibitor plasmids (miR-inhibitor group); (3) NEAT1 shRNA and NC inhibitor plasmids (sh-NEAT1\&NC-inhibitor group); (4) NEAT1 shRNA and miR-193a inhibitor plasmids (shNEAT1\&miR-inhibitor group). Subsequently, lnc-NEAT1 expression was detected by qPCR at $24 \mathrm{~h}$, cell proliferation was detected by CCK- 8 at $0 \mathrm{~h}, 24 \mathrm{~h}, 48 \mathrm{~h}$ and $72 \mathrm{~h}$, cell apoptosis rate was detected by $\mathrm{AV} / \mathrm{PI}$ at $72 \mathrm{~h}$.

qPCR

qPCR was used to assess the lnc-NEAT1 expression in cells. Firstly, total RNA was extracted from cells using TRIzol solution (Invitrogen, USA); Secondly, RNA was reversely transcribed using PrimeScript RT reagent (Takara, Japan); 
Thirdly, qPCR was conducted using the One Step SYBR PrimeScript RT-PCR Kit (Takara, Japan). Besides, GAPDH was applied as internal reference in qPCR assay, and lnc-NEAT1 expression was calculated by $2^{-\triangle \Delta C t}$ formula. Primers used for qPCR were presented in Table 1.

\section{CCK8}

Cell proliferation ability was assessed by Cell Counting Kit-8 (Dojindo, Japan) at $0 \mathrm{~h}, 24 \mathrm{~h}, 48 \mathrm{~h}$ and $72 \mathrm{~h}$ following the instructions of manufacture. Cells were washed by PBS, and then $10 \mu \mathrm{L}$ CCK- 8 and $90 \mu \mathrm{L}$ serum free medium were added. After incubation for $2 \mathrm{~h}$ under the condition of $95 \%$ air plus $5 \% \mathrm{CO} 2$ at $37^{\circ} \mathrm{C}$, cell proliferation rate was assessed according to the optical density (OD) value $(450 \mathrm{~nm})$ detected by Microplate reader (BioTek, USA). Cell Counting Kit-8 (Dojindo, Japan).

\section{Av/pi}

Cell apoptosis rate was assessed by AV/PI assay, and Dead Cell Apoptosis Kit with Annexin V Alexa Fluor ${ }^{\text {Tax }} 488$ \& Propidium Iodide (Invitrogen, USA) was used. Firstly, 0.25\% Tyrisin was used to trypsinize the cells, followed by the termination with serum free medium. Secondly, centrifugation (1800 rpm, $3 \mathrm{~min}$ ) was performed, and $100 \mu \mathrm{L}$ suspension was prepared after the supernatant was discarded. Thirdly, $2 \mu \mathrm{L}$ AV was added in darkness with incubation for 15 mins, and then $1 \mu \mathrm{L}$ PI was added to cells. Cell apoptosis rate was evaluated by flow cytometry (FCM) (Beckman, USA).

\section{Statistics}

SPSS 19.0 Software (IBM, USA) and GraphPad 6.01 Software (GraphPad, USA) were applied for statistics and graphs in this study. Data were presented as mean \pm standard deviation. Comparison among groups was detected by One-Way ANOVA test followed by multiple comparisons test (Dunnett-t test), and comparison between two groups was detected by t test. $P<0.05$ was considered significant.

\section{Results}

Validation of myocardial I/R injury model establishment Cell apoptosis rate was remarkably higher in OGD/R group compared to normal group $(P<0.001)$, suggesting the successful establishment of myocardial $\mathrm{I} / \mathrm{R}$ injury model (Fig. 1a-b). Moreover, qPCR was performed to assess the
lnc-NEAT1 expression between myocardial I/R injury model and normal myocardial cells, which displayed that lncNEAT1 relative expression was elevated in OGD/R group compared to normal group (Fig. 2$)(P<0.01)$.

\section{Effect of Inc-NEAT1 downregulation on myocardial I/R injury model}

Lnc-NEAT1 expression was greatly lower in OGD/R\&shNEAT1 group compared to OGD/R\&sh-NC group, indicating the successful transfection of lnc-NEAT1 shRNA plasmids (Fig. 3$)(P<0.001)$. Cell proliferation rate was increased in OGD/R\&sh-NEAT1 group at $48 \mathrm{~h}(P<0.01)$ and $72 \mathrm{~h}(\mathrm{P}<0.01)$ compared to OGD/R\&sh-NC group (Fig. 4a). For cell apoptosis rate, it was lower in OGC/ R\&sh-NEAT1 group compared to OGD/R\&sh-NC group (Fig. 4b-c) $(P<0.01)$. These results suggested that downregulation of lnc-NEAT1 enhanced cell proliferation and repressed cell apoptosis in I/R injury H9c2 cells.

\section{Expressions of candidate target miRNAs of Inc-NEAT1}

In order to further explore the detailed mechanism of lncNEAT1 in myocardial I/R injury, expressions of candidate target miRNAs of lnc-NEAT1 were evaluated (Fig. 5). And we observed that miR-193a was downregulated while miR-182 and miR-141 were upregulated in OGD/R\&sh$\mathrm{NC}$ group compared to normal\&sh-NC group, indicating that these miRNAs play a role in the myocardial $I / R$ injury. Most importantly, miR-193a expression was increased but miR-182 and miR-141 expressions were undifferentiated in OGC/R\&sh-NEAT1 group compared with OGD/R\&sh-NC group, suggesting that only miR193a was targeted by lnc-NEAT1 in I/R injury H9c2 cells.

\section{Rescue experiments}

In order to identify whether lnc-NEAT1 regulates H9c2 cells functions through targeting miR-193a, rescue experiment was performed, which disclosed that miR-193a expression was reduced in $\mathrm{OGD} / \mathrm{R} \& \mathrm{miR}$-inhibitor group compared to OGD/R\&NC-inhibitor group $(P<0.01)$, while it was lower in sh-NEAT1\&miR-inhibitor group compared to shNEAT1\&NC-inhibitor group at $24 \mathrm{~h}$, indicating the successful transfections of miR-193a inhibitor plasmids in I/R injury H9c2 cells (Fig. 6) $(P<0.01)$. In the followed CCK8 assay, cell proliferation rate was reduced in OGD/R\&miR-inhibitor

Table 1 Primers in $\mathrm{qPCR}$

\begin{tabular}{|c|c|c|}
\hline Gene & Forward Primer & Reverse Primer \\
\hline NEAT1 & 5' GCCAGTGTGAGTCCTAGCATTG 3' & 5' ACTTCCTCCTCCTAAGCCTCTG 3' \\
\hline miR-193a & 5' ACACTCCAGCTGGGACTGGGACTTTGTAGGCCA 3' & 5' TGTCGTGGAGTCGGCAATTC 3' \\
\hline miR-182 & 5' ACACTCCAGCTGGGTTTGGCAATGGTAGAACT 3' & 5' TGTCGTGGAGTCGGCAATTC 3' \\
\hline miR-141 & 5' ACACTCCAGCTGGGTAACACTGTCTGGTAAAG 3' & 5' TGTCGTGGAGTCGGCAATTC 3' \\
\hline U6 & 5' CGCTTCGGCAGCACATATACTA 3' & 5' ATGGAACGCTTCACGAATTTGC 3' \\
\hline GAPDH & 5' GAGTCCACTGGCGTCTTCAC 3' & 5' ATCTTGAGGCTGTTGTCATACTTCT 3 \\
\hline
\end{tabular}



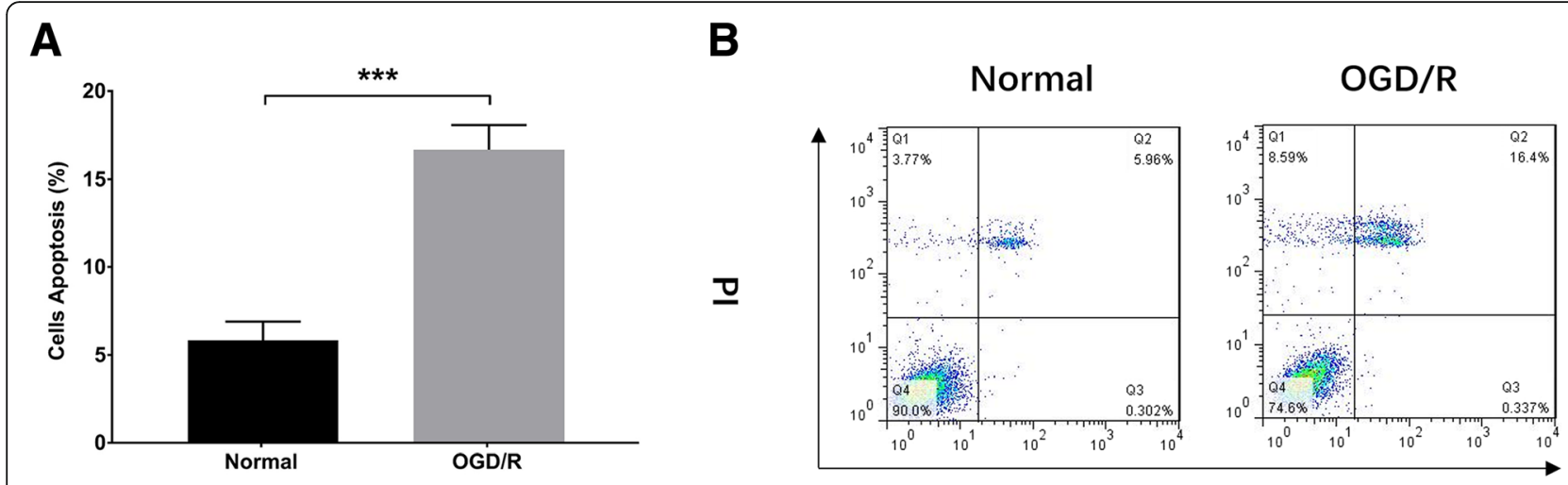

\section{AV}

Fig. 1 Cell apoptosis rate in OGD/R group and normal group. Cell apoptosis rate was elevated in OGD/R group compared to normal group (a-b). Comparison between two groups was assessed by t test. OGD/R, oxygen and glucose deprivation followed by reperfusion. $P<0.05$ was considered significant. ${ }^{* * *} P<0.001$

group compared to OGD/R\&NC-inhibitor group at $48 \mathrm{~h}$ $(P<0.05)$ and $72 \mathrm{~h}(P<0.01)$, and it decreased in OGD/ R\&sh-NEAT1\&miR-inhibitor group compared to OGD/ R\&sh-NEAT1\&NC-inhibitor group at $48 \mathrm{~h}(P<0.05)$ and 72 $\mathrm{h}(P<0.01)$ (Fig. 7a), suggesting that downregulation of lncNEAT1 promoted cell proliferation via targeting miR-193a in $\mathrm{I} / \mathrm{R}$ injury H9c2 cells. Furthermore, cell apoptosis rate detected by AV/PI assay was increased in OGD/R\&miR-inhibitor group compared to OGD/R\&NC-inhibitor group at $72 \mathrm{~h}$ $(P<0.01)$, and it was elevated in OGD/R\&sh-NEAT1\&miRinhibitor group compared to OGD/R\&sh-NEAT1\&NC-inhibitor group at $72 \mathrm{~h}$ (Fig. $7 \mathrm{~b}-\mathrm{c})(P<0.01)$, suggesting that downregulation of lnc-NEAT1 inhibited cell apoptosis through targeting miR-193a in I/R injury H9c2 cells.

\section{Discussion}

In this study, we found that: (1) lnc-NEAT1 expression was higher in $\mathrm{I} / \mathrm{R}$ injury $\mathrm{H} 9 \mathrm{c} 2$ cells compared to normal

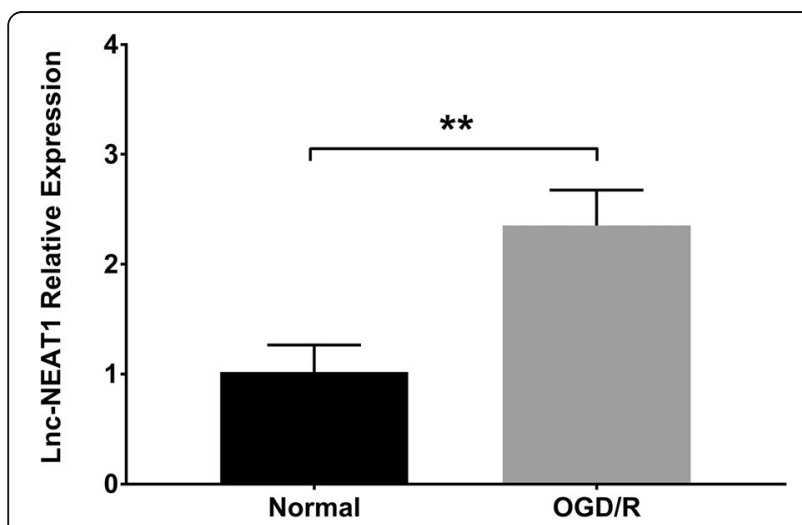

Fig. 2 Lnc-NEAT1 expression in OGD/R group and normal group. Compared with normal group, Inc-NEAT1 expression was raised in OGD/R group. OGD/R, oxygen and glucose deprivation followed by reperfusion. $\mathrm{P}<0.05$ was considered significant. ${ }^{* *} p<0.01$
H9c2 cells, and down-regulation of lnc-NEAT1 promoted cell proliferation and inhibited apoptosis in $\mathrm{I} / \mathrm{R}$ injury H9c2 cells; (2) rescue experiment revealed that down-regulation of lnc-NEAT1 enhanced cell proliferation and repressed cell apoptosis via targeting miR-193a in $\mathrm{I} / \mathrm{R}$ injury $\mathrm{H} 9 \mathrm{c} 2$ cells.

LncRNAs acts as new modulators involving in physiological processes to maintain cellular and tissue homeostasis, and parts of their dysregulated expressions devote into the onset and progression of multiple pathological conditions, including the pathophysiology of myocardial I/R injury [18]. For instance, lncRNA Reprogramming (ROR) reduces cells viability and promotes cell apoptosis

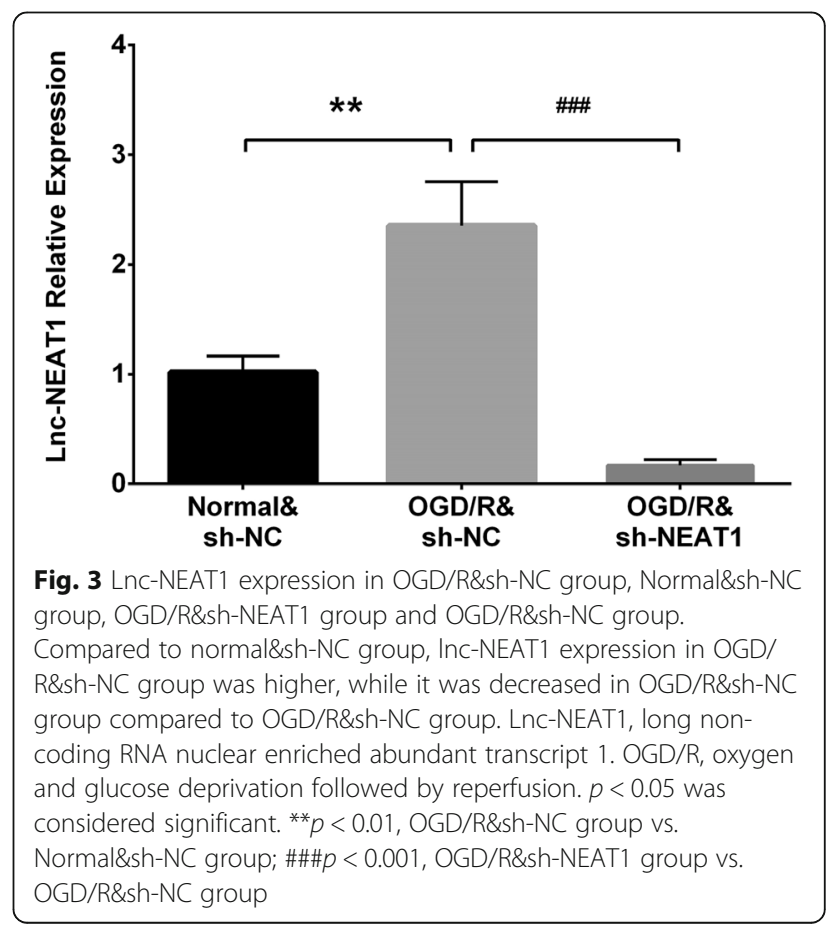




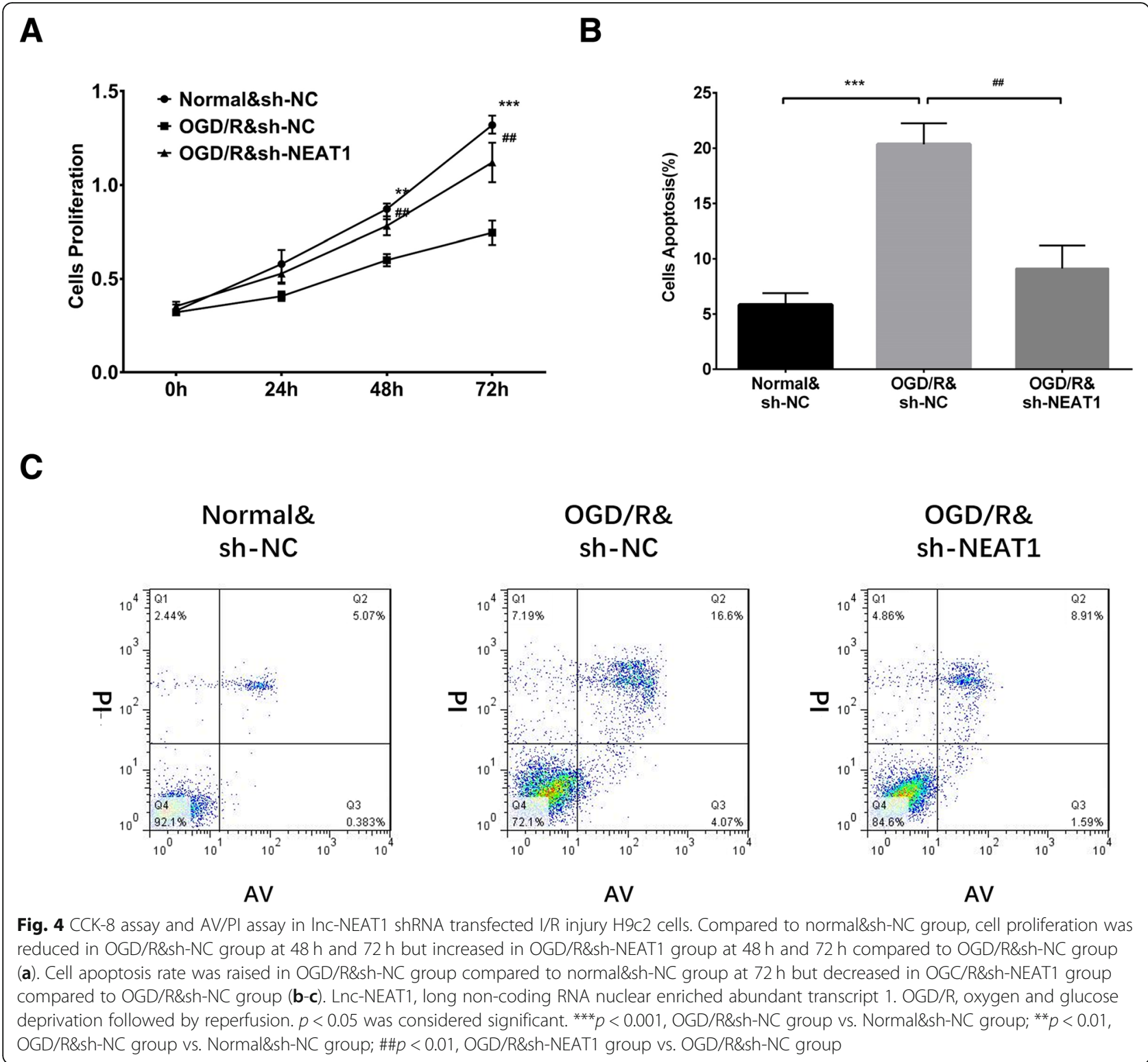

through improving p38 phosphorylation, inhibiting p38/ MAPK and rescuing lncRNA-ROR-induced cell injury in H9c2 cells [18]. Besides, lncRNA urothelial carcinomaassociated 1 (UCA1) contributes to cardiomyocyte apoptosis via suppressing p27 level in rat cardiac ischemia reperfusion injury [19]. Another study displays that downregulation of IncRNA-MALAT1 enhances cardiomyocyte apoptosis by targeting miR-145/Bnip3 pathway. Thus, these evidences reveal that lncRNAs involve into the pathological processes of the myocardial I/R injury.

For lnc-NEAT1, it has been reported to be abnormally expressed in various diseases, and it could affect cell proliferation, cells migration and cells invasion through regulating multiple genes or pathways such as Ras-mitogen-activated protein kinase (MAPK), Wnt/ $\beta$-catenin pathway and miR-
377-3p-E2F3 pathways [9, 20]. For example, a study conducted by Sun et al. displays that lnc-NEAT1 promotes cell proliferation in non-small cell lung cancer, and another study discloses that lnc-NEAT1 enhances cell proliferation, invasion and migration in endometrial endometrioid adenocarcinoma cells $[20,21]$. Furthermore, a review reveals that neutrophils play important role in $I / R$ injury due to their proximity at endothelium and other inflammatory cells at the vascular interface [22]. Once neutrophil adhesion is established, neutrophils infiltrate the ischemic tissues, and neutrophil infiltration into the infarcted area indicates the generation of reactive oxygen species (ROS) and proteolytic enzymes that injure the surrounding cardiomyocytes. Additionally, macrophages show both inhibitory actions by secreting mediators suppressing inflammation and pro- 
A

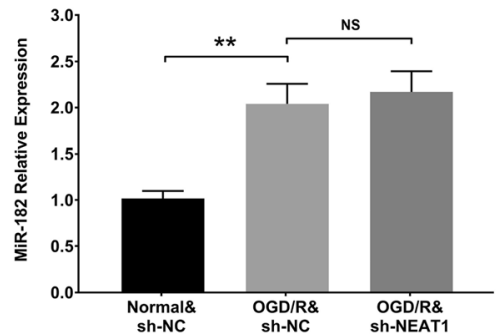

B

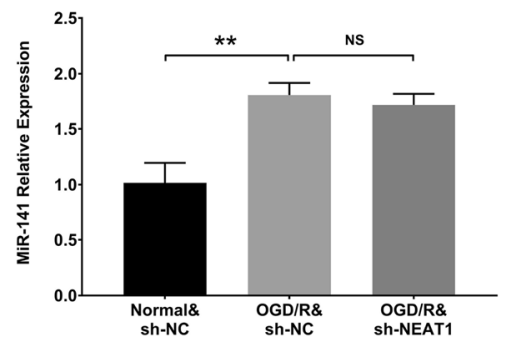

C

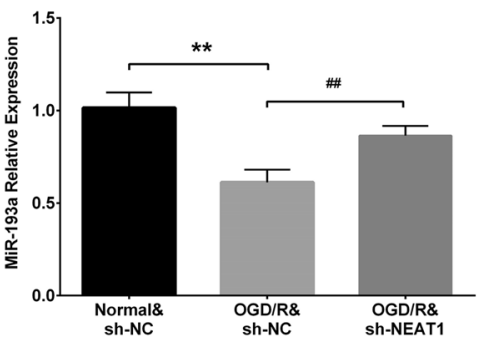

Fig. 5 Prediction and detection of target miRNAs of Inc-NEAT1. Both miR-182 expression and miR-141 expression were elevated in OGD/R\&sh-NC group compared to normal\&sh-NC group at $24 \mathrm{~h}$, while no difference of miR-182 expression or miR-141 expression was found between OGC/ R\&sh-NEAT1 group and OGD/R\&sh-NC group (a-b). Furthermore, miR-193a expression was reduced at $24 \mathrm{~h}$ in OGD/R\&sh-NC group compared to normal\&sh-NC group but raised in OGC/R\&sh-NEAT1 group compared to OGD/R\&sh-NC group at 24 h. Lnc-NEAT1, long non-coding RNA nuclear enriched abundant transcript 1. OGD/R, oxygen and glucose deprivation followed by reperfusion. $P<0.05$ was considered significant. ${ }^{* *}, p<0.01$, OGD/R\&sh-NC group vs. Normal\&sh-NC group; \#\#, $p<0.01$, OGD/R\&sh-NEAT1 group vs. OGD/R\&sh-NC group; NS, no significant difference

resolving actions in order to remove inflammatory leucocytes during postinfarction inflammation [22]. For lnc-NEAT1, one study shows that lnc-NEAT1 presents higher expression in neutrophils compared to other immune cells and lncNEAT1 may derive from metastatic tissues and neutrophils, meanwhile, lnc-NEAT1 promotes ox-LDL-induced inflammation and oxidative stress in macrophages [23, 24]. Although these previous studies reveal that lnc-NEAT1 serves as an oncogene in various cancers through promoting cell proliferation and inhibiting cell apoptosis, moreover, lnc-NEAT1 may present influences in neutrophils and macrophages, which are inflammatory cells involved in the myocardial I/R injury, little

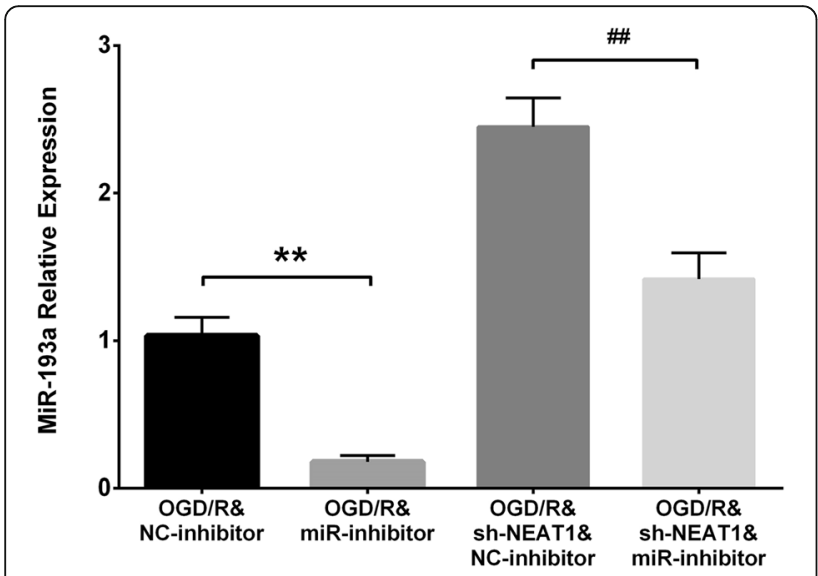

Fig. 6 Measurement of miR-193a after transfected with NEAT1 shRNA and miR-193a inhibitor plasmids. Compared to OGD/R\&NCinhibitor group, miR-193a expression was lower in OGD/R\&miRinhibitor group, whereas it was decreased in sh-NEAT1\&miR-inhibitor group compared to sh-NEAT1\&NC-inhibitor group at $24 \mathrm{~h}$. LncNEAT1, long non-coding RNA nuclear enriched abundant transcript 1. OGD/R, oxygen and glucose deprivation followed by reperfusion. $p<0.05$ was considered significant. ${ }^{* *} p<0.01, \mathrm{OGD} /$ R\&miR-inhibitor group vs. OGD/R\&NC-inhibitor group; \#\#p $<0.01$, OGD/R\&sh-NEAT1\&miR-inhibitor group vs.

OGD/R\&sh-NEAT1\&NC-inhibitor group is known about the effect of lnc-NEAT1 on cells activities in cardiovascular diseases, particularly myocardial I/R injury. In this study, we detected lnc-NEAT1 expression in myocardial $\mathrm{I} / \mathrm{R}$ injury cells as well as normal myocardial cells, and we found that lnc-NEAT1 expression was elevated in myocardial I/R injury cells compared to normal cells, moreover, downregulation of lnc-NEAT1 increased myocardial I/R injury cell proliferation but reduced cell apoptosis, indicating that lncNEAT1 might play an unfavorable role in protecting against myocardial ischemia, and the downregulation of lnc-NEAT1 might shed a light to the treatment of myocardial ischemia due to its promotion of myocardial cell proliferation and inhibition of cell apoptosis. In addition, only the rat cardiac muscle cell line $\mathrm{H} 9 \mathrm{c} 2$ was available for this study due to the scarcity in diversity of myocardial cells in China, which resulted in the lack of verification in other cell lines, and similar limitations could be observed in some previous studies that focus on myocardial ischemia/reperfusion (I/R) injury $[25,26]$.

MicroRNA-193a (miR-193a), which is embedded in a CpG island, has been identified as a tumor suppressive miRNA in several cancers such as oral carcinoma, lung cancer and colorectal cancer [27-33]. Apart from cancers, it is disclosed that miR-193a is downregulated in intestinal inflammation in colitis, and upregulation of miR-193a promotes anti-inflammatory activities via targeting apoptotic pathways death receptor-6 (DR6) [34]. Hence, these previous evidences reveal that miR-193a influence cells activities in diverse diseases, thereby devoting to these disease developments and progressions, whereas few evidences of miR-193a in affecting I/R injury myocardial cells have been reported. In this present study, we found that downregulation of lnc-NEAT1 promoted cell proliferation and repressed apoptosis by targeting miR-193a, furthermore, we conducted rescue experiments, which revealed that down-regulating lncNEAT1 affected cell proliferation and cell apoptosis through regulating miR-193a in I/R injury $\mathrm{H} 9 \mathrm{c} 2$ cells, 


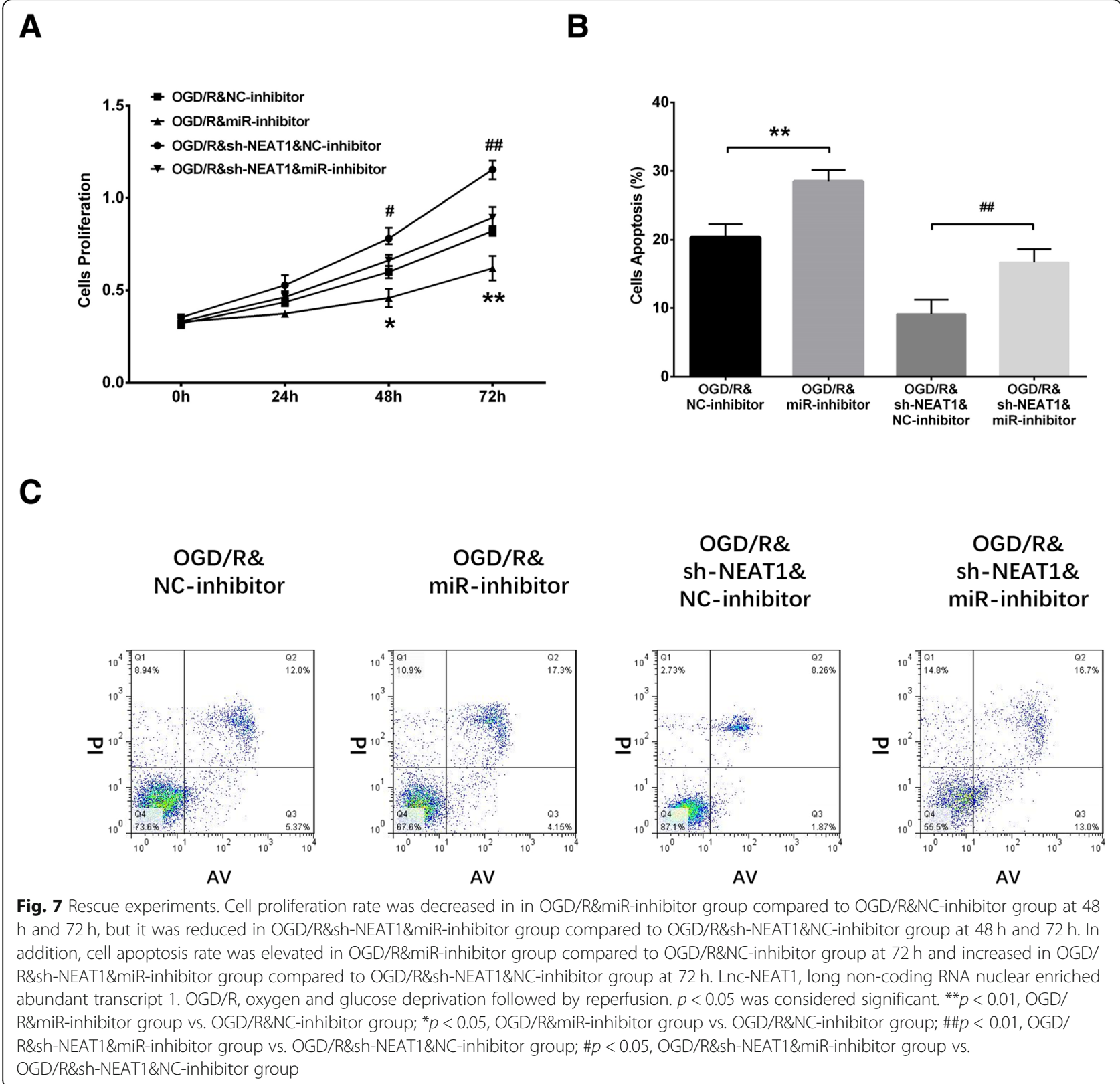

and these results might provide indications for further exploring mechanisms of myocardial I/R injury. Considering the over-expression of lnc-NEAT1 in myocardial $I / R$ injury cells, and the promotion of myocardial cell proliferation as well as inhibition of cell apoptosis by lnc-NEAT1 downregulation, lnc-NEAT1 had the potential to applied in better stratification of patients suffering from myocardial $I / R$ injury and be a novel target for treatment of myocardial $I / R$ injury. Further study is needed to verify our findings as well as investigate the role of lnc-NEAT1 in myocardial I/R injury with clinical practices.

\section{Conclusions}

lnc-NEAT1 is overexpressed in myocardial I/R injury cells compared to normal myocardial cells, and downregulation of lnc-NEAT1 enhances cell proliferation while inhibits cell apoptosis through targeting miR-193a in $\mathrm{I} / \mathrm{R}$ injury $\mathrm{H} 9 \mathrm{c} 2$ cells.

\section{Abbreviations}

DMEM: Dulbecco's Modified Eagle's Medium; DR6: death receptor-6; FBS: Fetal Bovine Serum; FCM: flow cytometry; I/R: ischemia/reperfusion; IncNEAT1: LncRNA nuclear enriched abundant transcript 1; IncRNAs: long noncoding RNAs; MAPK: Ras-mitogen-activated protein kinase; miR-

193a: MicroRNA-193a; OGD/R: oxygen and glucose deprivation followed by reperfusion; ROR: Reprogramming; UCA1: urothelial carcinoma-associated 1 


\section{Acknowledgements}

Not applicable.

\section{Authors' contributions}

LYR, SSC and HY provided the hypothesis, and handled funding and supervision. JWL, PH and WS collected and analyzed the data. All authors drafted and revised the manuscript. All authors approved the final version to be published.

\section{Funding}

This study was supported by the project of "Effects of the AGEs_RAGE signaling pathway on mediated by hydroxysafflower flavin A on cerebral inflammatory responses to hepatic ischemia reperfusion" (2018CFC807).

\section{Availability of data and materials}

All relevant data is presented in the manuscript and supporting material.

\section{Ethics approval and consent to participate}

Not applicable.

\section{Consent for publication}

Not applicable.

\section{Competing interests}

The authors declare that they have no competing interests.

\section{Author details}

'Department of Anesthesiology, The Central Hospital of Wuhan, Tongji Medical College, Huazhong University of Science and Technology, 26, Shengli Street, Jiang'an District, Wuhan 430014, China. ${ }^{2}$ Key Laboratory for Molecular Diagnosis of Hubei Province, The Central Hospital of Wuhan, Tongji Medical College, Huazhong University of Science and Technology, 26, Shengli Street, Jiang'an District, Wuhan 430014, China.

Received: 7 March 2019 Accepted: 28 May 2019

Published online: 07 August 2019

\section{References}

1. Sacks FM, Lichtenstein AH, Wu JHY, Appel L, Creager MA, Kris-Etherton PM, Miller M, Rimm EB, Rudel LL, Robinson JG, et al. Dietary fats and cardiovascular disease: a presidential advisory from the American Heart Association. Circulation. 2017:136(3):e1-e23.

2. Jiang L, Krumholz HM, Li X, Li J, Hu S. Achieving best outcomes for patients with cardiovascular disease in China by enhancing the quality of medical care and establishing a learning health-care system. Lancet. 2015;386(10002):1493-505.

3. Guzman E, Molina J. The predictive utility of the plant phylogeny in identifying sources of cardiovascular drugs. Pharm Biol. 2018;56(1):154-64.

4. Shecterle LM, Terry KR, St Cyr JA. Potential clinical benefits of D-ribose in ischemic cardiovascular disease. Cureus. 2018;10(3):e2291.

5. Lejay A, Fang F, John R, Van JA, Barr M, Thaveau F, Chakfe N, Geny B, Scholey JW. Ischemia reperfusion injury, ischemic conditioning and diabetes mellitus. J Mol Cell Cardiol. 2016;91:11-22.

6. Neri M, Riezzo I, Pascale N, Pomara C, Turillazzi E. Ischemia/reperfusion injury following acute myocardial infarction: a critical issue for clinicians and forensic pathologists. Mediat Inflamm. 2017;2017:7018393.

7. Cadenas S. ROS and redox signaling in myocardial ischemia-reperfusion injury and cardioprotection. Free Radic Biol Med. 2018:117:76-89.

8. Long B, Li N, Xu XX, Li XX, Xu XJ, Guo D, Zhang D, Wu ZH, Zhang SY. Long noncoding RNA FTX regulates cardiomyocyte apoptosis by targeting miR29b-1-5p and Bc|212. Biochem Biophys Res Commun. 2018;495(1):312-8.

9. Wang J, Zhao X, Guo Z, Ma X, Song Y, Guo Y. Regulation of NEAT1/miR-214 $3 p$ on the growth, migration and invasion of endometrial carcinoma cells. Arch Gynecol Obstet. 2017;295(6):1469-75.

10. Engreitz JM, Ollikainen N, Guttman M. Long non-coding RNAs: spatial amplifiers that control nuclear structure and gene expression. Nat Rev Mol Cell Biol. 2016;17(12):756-70.

11. Rinn JL, Chang HY. Genome regulation by long noncoding RNAs. Annu Rev Biochem. 2012;81:145-66.

12. Kawaguchi T, Hirose T. Chromatin remodeling complexes in the assembly of long noncoding RNA-dependent nuclear bodies. Nucleus (Calcutta). 2015;6(6):462-7.
13. Chujo T, Yamazaki T, Hirose T. Architectural RNAs (arcRNAs): a class of long noncoding RNAs that function as the scaffold of nuclear bodies. Biochim Biophys Acta. 2016;1859(1):139-46.

14. Yu B, Shan G. Functions of long noncoding RNAs in the nucleus. Nucleus (Calcutta). 2016;7(2):155-66

15. Zhong J, Jiang L, Huang Z, Zhang H, Cheng C, Liu H, He J, Wu J, Darwazeh $\mathrm{R}$, Wu Y, et al. The long non-coding RNA Neat1 is an important mediator of the therapeutic effect of bexarotene on traumatic brain injury in mice. Brain Behav Immun. 2017:65:183-94.

16. Kesherwani V, Shahshahan HR, Mishra PK Cardiac transcriptome profiling of diabetic Akita mice using microarray and next generation sequencing. PLoS One. 2017;12(8):e0182828

17. Zhang $H$, Cai $Y$, Zheng $L$, Zhang Z, Lin $X$, Jiang $N$. Long noncoding RNA NEAT1 regulate papillary thyroid cancer progression by modulating miR129-5p/KLK7 expression. J Cell Physiol. 2018;233(10):6638-48.

18. Zhang W, Li Y, Wang P. Long non-coding RNA-ROR aggravates myocardial ischemia/reperfusion injury. Braz J Med Biol Res. 2018;51(6):e6555.

19. Liu Y, Zhou D, Li G, Ming X, Tu Y, Tian J, Lu H, Yu B. Long non coding RNAUCA1 contributes to cardiomyocyte apoptosis by suppression of p27 expression. Cell Physiol Biochem. 2015;35(5):1986-98.

20. Sun C, Li S, Zhang F, Xi Y, Wang L, Bi Y, Li D. Long non-coding RNA NEAT1 promotes non-small cell lung cancer progression through regulation of miR-377-3p-E2F3 pathway. Oncotarget. 2016;7(32):51784-814.

21. Li Z, Wei D, Yang C, Sun H, Lu T, Kuang D. Overexpression of long noncoding RNA, NEAT1 promotes cell proliferation, invasion and migration in endometrial endometrioid adenocarcinoma. Biomed Pharmacother. 2016;84:244-51.

22. Bonaventura A, Montecucco F, Dallegri F. Cellular recruitment in myocardial ischaemia/reperfusion injury. Eur J Clin Investig. 2016;46(6):590-601.

23. Wu Y, Yang L, Zhao J, Li C, Nie J, Liu F, Zhuo C, Zheng Y, Li B, Wang Z, et al. Nuclear-enriched abundant transcript 1 as a diagnostic and prognostic biomarker in colorectal cancer. Mol Cancer. 2015;14:191.

24. Chen DD, Hui LL, Zhang XC, Chang Q. NEAT1 contributes to ox-LDLinduced inflammation and oxidative stress in macrophages through inhibiting miR-128. J Cell Biochem. 2018.

25. Chen L, Zhang D, Yu L, Dong H. Targeting MIAT reduces apoptosis of cardiomyocytes after ischemia/reperfusion injury. Bioengineered. 2019;10(1):121-32.

26. Liu DW, Zhang YN, Hu HJ, Zhang PQ, Cui W. Downregulation of microRNA199a5P attenuates hypoxia/reoxygenationinduced cytotoxicity in cardiomyocytes by targeting the HIF1alphaGSK3betamPTP axis. Mol Med Rep. 2019;19(6):5335-44.

27. Ling Z, Wang X, Tao T, Zhang L, Guan H, You Z, Lu K, Zhang G, Chen S, Wu J, et al. Involvement of aberrantly activated HOTAIR/EZH2/miR-193a feedback loop in progression of prostate cancer. J Exp Clin Cancer Res. 2017;36(1):159.

28. Kozaki K, Imoto I, Mogi S, Omura K, Inazawa J. Exploration of tumorsuppressive microRNAs silenced by DNA hypermethylation in oral cancer. Cancer Res. 2008:68(7):2094-105.

29. Liang H, Liu M, Yan X, Zhou Y, Wang W, Wang X, Fu Z, Wang N, Zhang S, Wang $Y$, et al. miR-193a-3p functions as a tumor suppressor in lung cancer by down-regulating ERBB4. J Biol Chem. 2015;290(2):926-40.

30. Pekow J, Meckel K, Dougherty U, Huang Y, Chen X, Almoghrabi A, Mustafi R, Ayaloglu-Butun F, Deng Z, Haider HI, et al. miR-193a-3p is a key tumor suppressor in ulcerative colitis-associated Colon Cancer and promotes carcinogenesis through upregulation of IL17RD. Clin Cancer Res. 2017;23(17):5281-91.

31. Gao XN, Lin J, Li YH, Gao L, Wang XR, Wang W, Kang HY, Yan GT, Wang LL, Yu L. MicroRNA-193a represses c-kit expression and functions as a methylation-silenced tumor suppressor in acute myeloid leukemia. Oncogene. 2011;30(31):3416-28.

32. Ma K, He Y, Zhang H, Fei Q, Niu D, Wang D, Ding $X, X u H$, Chen $X$, Zhu J. DNA methylation-regulated miR-193a-3p dictates resistance of hepatocellular carcinoma to 5 -fluorouracil via repression of SRSF2 expression. J Biol Chem. 2012:287(8):5639-49.

33. Heller G, Weinzierl M, Noll C, Babinsky V, Ziegler B, Altenberger C, Minichsdorfer C, Lang G, Dome B, End-Pfutzenreuter A, et al. Genome-wide miRNA expression profiling identifies miR-9-3 and miR-193a as targets for DNA methylation in nonsmall cell lung cancers. Clin Cancer Res. 2012;18(6):1619-29.

34. Alghetaa H, Mohammed A, Sultan M, Busbee P, Murphy A, Chatterjee S, Nagarkatti M, Nagarkatti P. Resveratrol protects mice against SEB-induced acute lung injury and mortality by miR-193a modulation that targets TGFbeta signalling. J Cell Mol Med. 2018;22(5):2644-55.

\section{Publisher's Note}

Springer Nature remains neutral with regard to jurisdictional claims in published maps and institutional affiliations. 